\title{
SERVIÇO DE ATENDIMENTO MÓVEL DE URGÊNCIA DO RIO GRANDE DO SUL
}

Magda Regina Dorr ${ }^{1}$

Gabriella Barbosa Nadas

Celia Sapin Duarte ${ }^{1}$

Cristiane Damiani Tomasi ${ }^{1}$

Lisiane Tuon ${ }^{1}$

\author{
http://orcid.org/0000-0003-0276-9350 \\ http://orcid.org/0000-0001-5558-1453 \\ http://orcid.org/0000-0001-5821-5432 \\ http://orcid.org/0000-0002-1813-765X \\ http://orcid.org/0000-0002-0809-0712
}

Objetivo: analisar os atendimentos realizados na Central de Regulação de Urgência/SAMU do Método: Estado do Rio Grande do Sul no período de 2016 e 2017, com foco no tempo resposta. estudo observacional, descritivo e retrospectivo com abordagem quantitativa. Foram coletados os dados sobre perfil do usuário, dia da semana, classificação de risco, tipo do agravo, tempo médio de atendimento às solicitações, quantitativo de desistência da solicitação e número de chamados não regulados por falta de equipes ou veículos. Resultados: o número de solicitações por motivos clínicos foi $53.8 \%$, destes 51,6\% foram média gravidade. A prevalência foi do sexo masculino e idade entre 18 e 29 anos. Em $84,7 \%$ dos chamados solicitou-se suporte básico. O tempo resposta total foi entre 30 minutos e 1 hora, maior que o recomendado de acordo com a média nacional, seja nas as zonas urbanas ou rurais. Conclusão: Após analisar os atendimentos, percebe-se que o pior problema dos atendimentos é o tempo resposta, sendo necessário diminuir o mesmo das solicitações.

Descritores: Indicadores; Central de Regulação Estadual; Serviços Médicos de Emergência; Administração de Serviços de Saúde.

\section{EMERGENCY MOBILE CARE SERVICE OF RIO GRANDE DO SUL}

Objective: to analyze the attendances performed at the Emergency Mobile Care Service of the State of Rio Grande do Sul in 2016 and 2017 , focusing in response time. Method: observational, descriptive and retrospective study with quantitative approach. Data were collected on user profile, day of the week, risk classification, type of grievance, average time to respond to requests, amount of withdrawal and number of calls not regulated due to lack of teams or vehicles. Results: The number of requests for clinical reasons was $53.8 \%$, of these $51.6 \%$ were medium severity. The prevalence was male and aged between 18 and 29 years. In $84.7 \%$ of the calls, basic support was requested. The total response time was between 30 minutes and 1 hour, longer than recommended according to the national average, whether in urban or rural areas. Conclusion: After analyzing the calls, it is clear that the worst problem with the calls is the response time, and it is necessary to decrease the same of the requests.

Descriptors: Indicators; Central of State Regulation; Emergency Medical Services; Health Services Administration.

\section{ANÁLISIS DE LLAMADAS DEL CENTRO DE REGULACIÓN EN RIO GRANDE DEL SUR}

Objetivo: analizar las asistencias realizadas en el Centro de Regulación de Emergencias / SAMU del Estado de Rio Grande do Sul en 2016 y 2017, centrándose en el tiempo de respuesta. Metodo: estudio observacional, descriptivo y retrospectivo con enfoque cuantitativo. Se recopilaron datos sobre el perfil del usuario, el día de la semana, la clasificación de riesgos, el tipo de queja, el tiempo promedio para responder a las solicitudes, la cantidad de retiros y la cantidad de llamadas no reguladas debido a la falta de equipos o vehículos. Resultados: El número de solicitudes por razones clínicas fue del 53.8\%, de estas 51.6\% fueron de gravedad media. La prevalencia fue masculina y de edades comprendidas entre 18 y 29 años. En el $84.7 \%$ de las llamadas, se solicitó soporte básico. El tiempo de respuesta total fue de entre 30 minutos y 1 hora, más de lo recomendado según el promedio nacional, ya sea en áreas urbanas o rurales. Conclusión: Después de analizar las llamadas, está claro que el peor problema con las llamadas es el tiempo de respuesta, y es necesario disminuir las mismas solicitudes.

Descriptores: Indicadores; Central de Regulación Estatal; Servicios Médicos de Urgencia; Administración de los Servicios de Salud.

IUniversidade do Extremo Sul Catarinense (UNESC)

Autor Correspondente: Lisiane Tuon - E-mail: ltb@unesc.net

Recebido:20/10/2019 - Aceito:30/03/2020 


\section{INTRODUÇÃO}

O Serviço de Atendimento Móvel de Urgência (SAMU) é um serviço do sistema único de saúde de atendimento pré-hospitalar móvel e é acionado por telefonia de discagem rápida (número 192), desta forma conhecido como SAMU 192.

No Brasil, as indagações que se referiam ao atendimento pré-hospitalar vieram a ser roteiros de debates na década de 1990 e o protótipo francês, com enfoque no médico regulador, foi empregado como referência norteadora. Nesse mesmo contexto, os policiais militares do corpo de bombeiros também deram início à qualificação em suporte pré-hospitalar, entretanto com base no sistema americano.(1) Em junho de 1998, foi divulgada a Portaria GM/MS 2923, que estabeleceu investimentos nos campos de Assistência Pré-hospitalar Móvel, Assistência Hospitalar, Centrais de Regulação de Urgências e Capacitação de Recursos Humanos. (1)

O SAMU é estruturado mediante uma rede de serviços regionalizada e hierarquizada para a atenção às urgências, a qual é regida pela Política Nacional de Atenção às Urgências. Sua logística se dá por meio de um processo de regulação que ocorre por intermédio de Centrais de Regulação de Urgência (CRU). ${ }^{(1)}$ As Centrais de Regulação de Urgência são observatórios privilegiados do Sistema de Saúde, pois tem o controle do gerenciamento dos fluxos de atenção às urgências, fazendo com que seja garantido o acesso universal aos afetados por agravos urgentes. São consideradas o eixo das políticas de urgência e emergência, e com base nisso, o Ministério da Saúde implementou a política pelo componente pré-hospitalar móvel.(1)

No estado do Rio Grande do Sul (RS), no municipio de Porto Alegre, o programa SAMU foi implantado em novembro de 1995. (2.3) Em 2003 o Estado do RS desenvolveu o projeto estadual para a implantação do serviço, assim, o programa implantado passou a se chamar SAMU/SALVAR-192, instituido pelo Decreto N 42.368, de 29/07/03. No RS atualmente existem cinco Centrais de Regulação Médica: Central Estadual, Central de Porto Alegre, Central de Pelotas, Central de Bagé, Central de Caxias do Sul e Vacaria. ${ }^{(4)}$

Historicamente, o nível de resposta do sistema de saúde às urgências e emergências é insuficiente, o que acarreta na superlotação das portas dos hospitais e pronto-socorro, mesmo quando a doença ou quadro clínico não é característica de um atendimento de emergência ou urgência. (5) Em complemento a isto, segundo Silva e Nogueira(b) ${ }^{(6)}$ no Brasil, entende-se como domínios deficientes do sistema de saúde, os de urgência e emergência, sendo caracterizados como áreas precárias do Sistema Único de Saúde (SUS), no qual as diretrizes de descentralização, regionalização e hierarquização encontram-se pouco implementadas.
Considerando a magnitude da política e sua implantação recente, o presente estudo tem como objetivo o serviço da Central de Regulação de Urgência/SAMU do Estado do Rio Grande do Sul e traçar o perfil dos atendimentos realizados no período de 2016 e 2017, com foco no tempo resposta de tais atendimentos.

\section{MÉTODO}

Estudo transversal, descritivo e retrospectivo com abordagem quantitativa. Iniciou-se o trabalho com 315.271 dados fornecidos pelo SAMU RS de 2016 a 2017, mas devido aos critérios de exclusão, eliminou-se 34.517 dados por serem menores de 18 anos e 5.879 por terem o tipo de gravidade classificada como "Orientações". Foram utilizados 280.754 dados no final.

O estudo foi elaborado a partir do banco de dados disponibilizado pela Central de Regulação Estadual, localizada na cidade de Porto Alegre, que é a principal e a maior central de Regulação do Rio Grande do Sul que onde também se situa uma regulação municipal. A Central Estadual regula 161 bases de atendimento, 38 de Suporte Avançado (AS) e 187 de Suporte Básico (SB), que atendem a 276 municípios distribuídos na região metropolitana e nas demais regiões de saúde. Esta central utiliza o sistema TRUE como armazenamento de dados. A população em estudo consiste nos usuários que fizeram chamadas telefônicas com informações pertinentes aos dados obtidos pelo referido SAMU/RS, no período de janeiro de 2016 a dezembro de 2017.

Os dados foram fornecidos pela gerência da CRU SAMU/ RS, conforme roteiro disponibilizado pelo sistema TRUE, em que foram coletados os dados sobre perfil do usuário, origem do chamado, horário de atendimento, classificação de risco, tipo e motivo do agravo, encaminhamentos médicos, tipo de veículo utilizado, chamados não regulados por falta de meios, ligações com desistência pelo solicitante.

Os critérios de inclusão são todos os registros das chamadas telefônicas com informações completas que aguardaram falar com o médico regulador. Os critérios de exclusão consistem nos registros das chamadas telefônicas que não possuem todas as informações necessárias para a pesquisa, ligações interrompidas e desistência de solicitações.

Para a análise estatística foi montado banco de dados a partir das informações fornecidas e processadas no software estatístico SPSS, versão 23.0 IBM®. As variáveis quantitativas foram apresentadas conforme a sua frequência relativa e sua porcentagem em relação ao total, conforme distribuição dos dados. Para análise de tabulação cruzada, utilizamos o índice de Kappa. Para todas as análises estatísticas, foi adotado o valor de $p<0,05$ para indicar diferença estatisticamente significativa. 
A pesquisa foi iniciada após aprovação pelo Comitê de Ética e Pesquisa em Seres Humanos da Universidade do Extremo Sul Catarinense e autorização do local onde foi realizada a pesquisa mediante apresentação do projeto e Carta de Aceite, tendo como base a Resolução 466/12 do Conselho Nacional de Saúde (CNS). A pesquisa foi aprovada pelo CEPParecer no:2.539.250.

Tabela 01: Perfil epidemiológico das vitimas do SAMU-RS 2016-2017, (Rio Grande do Sul ,2019)

\begin{tabular}{|ll}
\hline Variável & $\mathbf{n}(\%)$ \\
\hline Faixa etária & \\
18-29 anos & $46730(16,7)$ \\
30-39 anos & $35527(12,7)$ \\
40-49 anos & $34860(12,4)$ \\
$50-59$ anos & $39626(14,1)$ \\
60-69 anos & $38815(13,8)$ \\
$70-79$ anos & $39155(14)$ \\
\hline Acima de 80 anos & $45569(16,3)$ \\
Total & $280282(100)^{*}$ \\
\hline Sexo & \\
\hline Masculino & $159285(56,7)$ \\
\hline Feminino & $121459(43,3)$ \\
\hline Total & $280744(100)^{\star *}$ \\
\hline
\end{tabular}

Fonte: Dados fornecidos através do banco de dados do SAMU. Cálculo da frequência relativa e sua porcentagem válida (\%) em relação ao total. *Dados omissos no sistema no total de $472 .{ }^{*}$ Dados omissos no sistema no total de 10.

$\mathrm{Na}$ tabela $\mathrm{O} 2$ podemos observar que a grande maioria das ligações telefônicas recebidas pela Central de Regulação de Urgência (CRU) foram de chamados onde a equipe se deslocou e não teve sucesso no atendimento. Tal evento pode dar-se por inúmeros detalhes, como em $32,8 \%$ o paciente foi removido por outrem, $19,6 \%$ o paciente recusou atendimento e $10,5 \%$ o solicitante cancelou o atendimento. Sabe-se que todos estes deslocamentos poderiam ter sido evitados se houvesse mais agilidade no serviço e se a população fosse melhor instruída.

Tabela 02: Incidente local nas ocorrências do SAMU-RS 2016-2017 e o número de atendimento por CRS do SAMU-RS 2016-2017, (Rio Grande do Sul,2019)

\section{Variável} $n(\%$

Enviar apoio à missão $126(0,6)$

Equipe já enviada 295(1,4)

Incidente desconhecido no local (trote) $649(3,1)$

Localização não encontrada/inexistente $310(1,5)$

Óbito 1999(9,4)

Paciente agressivo- equipe solicita cancelamento 216(1)

Paciente não está no local 2377(11,2)

Paciente recusa atendimento $4171(19,6)$

Paciente recusa hospitalização $1528(7,2)$

Paciente removido por outrem $6972(32,8)$

Paciente removido pela policia militar

217(1)

SA

$1(0)$

SB

6(0)

Solicitante cancelou $\mathrm{o}$ atendimento

$2225(10,5)$

Troca de equipe

$135(0,6)$

Total

$21227(100)^{*}$

\section{Número de atendimento por CRS}

$n(\%)$

1aㅡ CRS- Porto Alegre (Metropolitano)

$66124(23,6)$

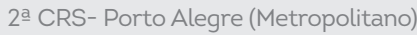

40348(14,4)

3a CRS- Pelotas (Sul)

$8106(2,9)$

4a CRS- Santa Maria (Centro-Oeste)

20938(7,5)

5믄 - Caxias do Sul (Serra)

$17586(6,3)$

6a CRS- Passo Fundo (Norte)

$16033(5,7)$

8 a CRS- Cachoeira do Sul (Vales)

$5579(2,0)$

9a CRS- Cruz Alta (Missioneira)

3424(1,2)

10ㅡㅡ CRS-Alegrete (Centro-Oeste)

$16639(5,9)$

11ㄹ CRS- Erechim (Norte)

4706(1,7)

12 a CRS- Santo Ângelo (Missioneira)

$11508(4,1)$

13a CRS- Santa Cruz do Sul (Vales)

$14726(5,2)$

14a CRS- Santa Rosa (Missioneira)

$6295(2,2)$

15a CRS- Palmeiras das Missões (Norte)

$5823(2,1)$

16a CRS- Lajeado (Vales)

$8943(3,2)$

17ạ CRS - Ijui (Missioneira)

$5141(1,8)$

18a CRS - Osório (Metropolitano)

$23150(8,2)$

19a CRS- Frederico Westphalen (Norte)

$5636(2,0)$

Total $280705(100)^{* *}$

Fonte: Dados fornecidos através do banco de dados do SAMU. Cálculo da frequência relativa e sua porcentagem válida (\%) em relação ao total. SA: Suporte Avançado; SB: Suporte Básico. *Dados omissos no sistema no total de 259527. ${ }^{* *}$ Dados omissos no sistema no total de 49 . 
Referente a gravidade comprovada conforme tabela 03 , foram classificados como média gravidade 135.025 (51,6\%), gravidade indeterminada $52.826(20,2 \%)$, baixa gravidade 46.214 (17,7\%), severa gravidade 15.375 (5,9\%), óbito 12.311 (4,7\%). Dos 280.754 chamados telefônicos considerados válidos, 76,9\% receberam despacho de ambulância. O despacho não ocorreu para a totalidade das ocorrências devido a dois motivos: a presença de ocorrências de baixo nível de gravidade que, segundo os critérios do SAMU, não apresentam necessidade de ambulância (16,3\%) e o cancelamento do chamado pelo solicitante (6,6\%), porque os pacientes já haviam sido removidos, seja por terceiros, meios próprios ou outros serviços, conforme tabela 03. A maneira pela qual as ocorrências percorrem as etapas do atendimento pré-hospitalar é diferente dependendo da prioridade atribuída a elas e pelo conceito de regulação médica das urgências utilizado no Brasil

Tabela 03: Trauma Score (TS) comprovado, gravidade comprovada, tipo de equipe e tempo resposta do SAMU-RS 2016-2017, (Criciúma,2019).

\begin{tabular}{|c|c|}
\hline Variável & $n(\%)$ \\
\hline \multicolumn{2}{|l|}{ TS comprovada } \\
\hline Clínico & $140741(53,8)$ \\
\hline Obstétrico & $3676(1,4)$ \\
\hline Psiquiátrico & $32331(12,3)$ \\
\hline Traumático & $85053(32,5)$ \\
\hline Total & $261801(100)^{\star}$ \\
\hline Gravidade comprovada & $n(\%)$ \\
\hline Indeterminada & $52826(20,2)$ \\
\hline Pequena & $46214(17,7)$ \\
\hline Média & $135025(51,6)$ \\
\hline Severa & $15375(5,9)$ \\
\hline Óbito & $12311(4,7)$ \\
\hline Total & $261751(100)^{\star *}$ \\
\hline Tipo de equipe & $n(\%)$ \\
\hline Aérea & $5(0)$ \\
\hline Indeterminada & $3(0)$ \\
\hline IR & $165(0,1)$ \\
\hline MT & $231(0,1)$ \\
\hline SA & $42447(15,1)$ \\
\hline SB & 237897(84,7) \\
\hline Total & $280748(100)^{\star * *}$ \\
\hline Tempo respos & $n(\%)$ \\
\hline 0-5 minutos & $32(0)$ \\
\hline 6-10 minutos & $80(0,1)$ \\
\hline $11-15$ minutos & $203(0,1)$ \\
\hline 16-20 minutos & $964(0,6)$ \\
\hline $21-30$ minutos & $11752(7,9)$ \\
\hline 30 minutos a $\mathrm{Ol}$ hora & $92856(62,4)$ \\
\hline Acima de $\mathrm{Ol}$ hora & $42843(28,8)$ \\
\hline Total & $148730(100)^{\star \star \star \star}$ \\
\hline
\end{tabular}

Fonte: Dados fornecidos através do banco de dados do SAMU. Cálculo da frequência relativa e sua porcentagem válida (\%) em relação ao total. *Dados omissos no sistema no total de 18953. **Dados omissos no sistema no total de 19003. ${ }^{* * *}$ Dados omissos no sistema no total de 6. TS: Trauma Score; IR:intervenção rapida ; MT:motolancia ; SA: Suporte Avançado; SB: Suporte Básico. ****

Dados omissos no sistema no total de 132024.

Observando-se o tempo de regulação dos chamados conforme tabela 03, fica entre 30 minutos e 1 hora em $62,4 \%$ das ocorrências e acima de 1 hora com 28,8\%, sabendo-se que este tempo pode ter a influência dos transportes, que na maioria das regiões os pacientes são encaminhados para destinos mais longos.

\section{DISCUSSÃO}

Os dados obtidos nessa pesquisa permitiram caracterizar a demanda e o processo de trabalho do SAMU/RS, trazendo elementos para a discussão sobre seu papel dentro do sistema de saúde do Estado.

O sexo masculino foi predominante na pesquisa com $56,7 \%$. Este resultado com predominância masculina também foi encontrado em estudo, onde a maioria dos atendimentos foi prestada a usuários do sexo masculino, perfazendo 64.754 pessoas (55,22\%), na faixa etária de 20 a 60 anos, correspondendo a 64.679 (55,62\%). ${ }^{(7)}$

O maior número de atendimentos ao sexo masculino pode se relacionar ao fato de homens frequentemente recorrerem aos serviços em virtude de doenças agudas e casos de agilização de doenças crônicas já instaladas. As mulheres buscam outros serviços da rede de atenção, como a Atenção Primária à Saúde (APS), para realizar ações de prevenção. Por outro lado, homens tendem a delongar a procura por atendimento e se mostram resistentes a procurar os serviços de prevenção e promoção à saúde.(3)

Encontramos 16,3\% de atendimentos acima de 80 anos. Dentre as condições de vulnerabilidade e risco levadas em consideração pelos médicos reguladores, está a faixa etária do paciente. O número de idosos no Brasil aumentou 700\% desde 1960 a 2008, e há a projeção de que será o sexto país do mundo em número de idosos em 2020.(2) Pacientes idosos compõe de 12 a $21 \%$ dos atendimentos em prontos socorros e utilizam mais frequentemente serviços de emergência que pacientes jovens. Eles possuem maior probabilidade de chegar ao pronto socorro por ambulância, de apresentarem maiores taxas de internação (33\% a 50\%) e de necessitarem de vaga de Unidade de Terapia Intensiva. ${ }^{(8,9)}$

A regulação apresenta-se, de início, fortemente associada ao nível pré-hospitalar móvel de urgência, inserida nas normas desse tipo de atenção - a Portaria No 824, de 24 de junho de 1999- como Regulação Médica das Emergências. ${ }^{(10)}$ Este, "em ação integrada com outros profissionais", comanda, orienta e inspeciona, presencialmente e 
à distância, como informam os protocolos de intervenção médica pré-hospitalar, a assistência às urgências. Reputa a presença de uma Central de Regulação, combinada a uma Central de Internações que possibilita ao médico regulador (MR) a orientação dos locais disponíveis e convenientes para as suas decisões frente às condições de urgência.

Das situações classificadas como Incidentes, em $32,8 \%$ delas o usuário havia sido removido em momento anterior à chegada da equipe na cena, o que pode indicar que o solicitante ou a família decidiram não aguardar a equipe do SAMU. Somado a isso, 10,5\% dos incidentes foram classificados como cancelados, o que também pode ser indicativo de desistência do atendimento. Cabe aqui ressaltar a dúvida do por que 10,5\% dos atendimentos foram cancelados, estes dados podem ser de mal atendimento da Central de Regulação de Urgência (CRU), Médico regulador não conseguiu orientar de forma adequada, os pacientes esperaram por muito tempo e resolveram não aguardar o SAMU, ou sabe-se também índice elevados de trotes no SAMU.

A decisão por não aguardar equipe e remover de outras formas pode estar apoiada na percepção do solicitante, família ou usuário que julgam demora na chegada da equipe. Para aqueles que aguardam pelo socorro, a sensação do tempo, invariavelmente parece ser superior ao tempo real, permeado pela angústia e ansiedade que fazem parecer ainda mais prolongado o tempo.(2) Isso acarreta em uma percepção ruim ao sistema público de saúde, tanto pelos pacientes quanto familiares, que preferem optar por não aguardar a equipe devido à demora, falta de confiança no sistema e sensação de abandono. Outros SAMU do Brasil usam a mesma classificação de incidente. Em Juazeiro (BA), identificou-se que em 2,8\% dos chamados feitos a esse serviço, os usuários haviam sido removidos por terceiros, enquanto que em Palmas (TO) $11 \%$ das saídas, não geraram atendimentos e os motivos foram classificados como trotes, cancelamentos ou remoção por terceiros. ${ }^{(11,12)}$

A Macro Metropolitana absorveu, conforme os dados quase metade dos atendimentos $(46,2 \%)$ devido ao maior número de municípios que ela abrange e, no total, maior número populacional. Cabe ressaltar, conforme estudo, a implantação das centrais reguladoras é de fundamental importância para a efetiva e eficiente prestação dos serviços, além disso, também salientam os autores, o ato de regular é prerrogativa do médico, altamente dependente das condições estruturais das CRU e do seu conhecimento e capacidade de mobilização dos recursos e regras cabíveis. ${ }^{(13)}$

Os chamados clínicos tiveram predominância com
140.741 (53,8\%) conforme tabela 03, seguidos dos traumáticos com 85,053 (32,5\%). A predominância de quadros clínicos indica pacientes e familiares com assistência na APS comprometida. É indispensável ressaltar que a ausência ou a dificuldade de acesso à assistência nas incumbências de atenção primária, a não reparabilidade dessa assistência, e a ausência de possibilidade da contra referência do enfermo para o prosseguimento ambulatorial, levam os usuários com queixas crônicas e sociais a buscarem por atendimentos em unidades de urgência como admissão primária do sistema de saúde.

Diversos estudos já demonstraram que as ocorrências classificadas com determinantes de baixa prioridade consistem de pacientes de baixa gravidade clínica e que não possuem condições tempo dependentes, apresentando baixa prioridade no despacho de ambulância. (2,3,11,13) Um estudo realizado num centro urbano avaliou as ocorrências graves e detectou que 99\% dessas ocorrências não apresentava critérios considerados de alta gravidade (como administração de medicamentos, transporte realizado com sirene ligada e luzes acesas, entre outros). (2) Estes estudos concluem, portanto, que os protocolos são seguros para essas ocorrências, ou seja, não são pacientes com gravidade clínica elevada. Nenhum deles, porém, concluem ou recomendam que estes casos não necessitam de atendimento pré-hospitalar.

Em relação aos atendimentos de urgência, o SAMU não está sendo ágil o suficiente segundo os critérios da população, que utiliza outros meios para resolver sua solicitação de ajuda ao SAMU. A fim de avaliar eficiência do sistema de Atendimento Pré Hospitalar (APH) prestado pelo SAMU RS, é necessário lançar um olhar crítico ao processo de trabalho da equipe que atua na CRU. Em Tocantins foi identificado que $11 \%$ das ocorrências não receberam despacho de ambulâncias por motivos de trote, cancelamento pelo solicitante, removido por bombeiros, policiais ou terceiros. ${ }^{(12)} \mathrm{O}$ estudo realizado em Santa Catarina, demonstrou que dentre os cinco motivos para o não despacho de ambulância, o mais significativo foi "remoção da vítima" (por terceiros, meios próprios, Corpo de Bombeiros ou pela Polícia Militar). ${ }^{(14)}$ Em Porto Alegre, 3,5\% das ambulâncias despachadas não encontraram o paciente no local, pois a vítima já havia sido removida. ${ }^{(2)}$

Nos atendimentos de urgência por unidades móveis predominaram as USB, com 84,7\%, conforme tabela 03 Estas unidades são equipadas para realizar o primeiro atendimento às urgências e contam com técnico de enfermagem e condutor/socorrista, que intervém por meio de medidas não-invasivas sob orientação de médicos das 
centrais de regulação. ${ }^{(15)}$

Foi possivel identificar na literatura internacional que os países desenvolvidos utilizam entre 8 e 20 minutos como tempo resposta ideal.(2) Diante da inviabilidade econômica de prover este tipo de resposta a totalidade das ocorrências, os países estabelecem que este tempo resposta deve ser direcionado a pacientes com condições clínicas que ameaçam a vida, com alto risco de morte (trauma grave, Parada Cardio Respiratória, Infarto Agudo do Miocárdio, Acidente Vascular Cerebral, entre outros). A medida utilizada são utilizadas as metas de 75\%, 90\% e $95 \%$ dos pacientes atendidos abaixo do tempo resposta estabelecido. ${ }^{(2,14)}$ Este é o caso do Reino Unido, onde a meta é atender $75 \%$ dos casos de alto risco dentro de 8 minutos e $95 \%$ dentro de 19 minutos. No SAMU Porto Alegre (SAMU POA), foram atendidas $75 \%$ das ocorrências consideradas severas em 23,8 minutos. $^{(2)}$

No SAMU POA, em estudo identificou-se que ele tem como tempo resposta mediana de 19 minutos e terceiro quartil de 2,1 horas para o total das ocorrências. ${ }^{(2)}$ Em relação a natureza das ocorrências, o SAMU POA teve como tempo resposta mediana de 18 minutos e terceiro quartil de 26 minutos para ocorrências traumáticas e 20 e 28 minutos para ocorrências clínicas (diferenças estatisticamente significativas). Apesar das ocorrências severas apresentarem um tempo resposta menor em relação as ocorrências de gravidade pequena e média, a diferença não foi estaticamente significativa. A similitude dos tempos resposta entre os níveis de gravidade sugere dois tipos de cenário: ou CRU do SAMU POA possui recursos suficientes para a demanda, fazendo com que não haja fila de espera para o despacho de ambulâncias, ou não há um critério de priorização evidente utilizado pelos médicos.

Neste interim, o SAMU, sem capacidade de arbitrar sobre as diferentes portas do sistema, inclui pacientes que se adequam a sua oferta: atendimento pré-hospitalar de qualidade a pacientes com risco iminente de morte e transporte qualificado de pacientes com necessidade de intervenções hospitalares. É questionável sua capacidade de responder às necessidades de saúde da população e atuar de forma integrada com a rede de atenção à saúde.

\section{Contribuição para a Prática}

Como contribuição para a prática do estudo, o tempo resposta contribui para que as Centrais de Regulação aperfeiçoem suas respostas aos chamados e planejem políticas que estruturem melhor os serviços de regula- ção. Ao trabalharem com o tempo resposta para atendimento de pessoas em risco, sem tal regulação, número suficiente de profissionais qualificados, sem a quantidade de veículos ideal e hospitais acessiveis com leitos suficientes, tem-se a fatalidade de vítimas por motivos de fragilidade da rede.

Se a cada três anos tivessem estudos de levantamento de dados do SAMU, saberíamos os pontos positivos e negativos, fragilidades e potencialidades dos atendimentos e demandas, funcionalidade do serviço e monitoramento. Com isso posto, teríamos como base esse estudo para melhorar e qualificar os serviços em prol ao benefício da população.

\section{Limitações do Estudo}

Uma das maiores limitações do estudo foi o banco de dados. Houve falta de dados, incoerência nas respostas, falta de padronização e inconsistência de informações. Falta um banco mais atualizado, mais fidedigno, no qual informações adequadas são necessárias para que se tenha melhores resultados com a equipe.

Sugere-se novo estudo que compare as centrais de regulação de outros estados com a do Rio Grande do Sul. Sugere-se também a possibilidade de realizar tal estudo de forma periódica, para que possa avaliar melhora ou declínio na qualidade do serviço. Além disso, sugere-se que pesquisas de tal caráter tenham incentivo do governo e seus resultados sejam de domínio público.

\section{CONCLUSÃO}

Por fim, conclui-se que a pesquisa cumpriu o objetivo a que se propôs, na medida em que expõe e analisa os atendimentos realizados pela CRU/SAMU do estado do RS, durante o período de 2016 e 2017. Dentre todos os dados levantados, o grande problema da Central de Regulação/RS é a questão do tempo resposta, sendo que sua falha acarreta em óbitos ou sequelas irreparáveis aos usuários. Conclui-se que tal central ainda não está preparada para trabalhar como atendimento de urgência e emergência e que medidas públicas, como maior investimento, mais equipes e melhora da estrutura física das ruas, devem ser tomadas diante tal situação para que seja fornecido um serviço adequado a população brasileira.

\section{CONTRIBUIÇÕES DOS AUTORES:}

Todos os autores responsáveis pelo presente artigo participaram das etapas de coleta de dados, elaboração e finalização do artigo. 


\section{REFERÊNCIAS}

1 Ministério da Saúde (BR). Secretaria de atenção à saúde, Departamento de atenção especializada, Regulação médica das urgências. Brasilia (DF), 2006 [citado em 2017 jul. 22]. Disponivel em: http://bvsms.Saude.gov.br/bvs/publicações/regulação_ medica_urgencias.pdf

2 Ciconet RM. Tempo resposta de um serviço de atendimento móvel de urgência. Tese (doutorado) -- Universidade Federal do Rio Grande do Sul, Escola de Enfermagem, Programa de Pós Graduação em Enfermagem, Porto Alegre, Rio Grande do Sul, Brasil, 2003 [citado em 2017 abr. 12]. Disponivel em: https://www.lume.ufrgs.br/bitstream/handle/10183/129481/000976890. pdf?sequence $=1$.

3 Oliveira EA. Otimização da localização das bases de operação do Serviço de Atendimento Móvel de Urgência - SAMU/BH através do uso de técnicas de geoprocessamento. Caminhos de Geografia jun/2008 [citado em 2017 jul. 13]; 9(26):133-45. Disponivel em: http://www.ig.ufu.br/revista/caminhos.html

4 Ministério da Saúde (BR). (Secretaria Estadual da Saúde do Rio Grande do Sul). Saúde e você, 2013 [citado 2013 ago. 19]. Disponivel em: http://www.saude.rs.gov.br/conteudo/394/?como_funciona_a_regula\%C3\%A7\%C3\%A30_do_SAMU\%3F

5 Ministério da Saúde (BR). Portaria no 2.048, de 5 de novembro de 2002. Aprova o regulamento técnico dos sistemas estaduais de urgência e emergência. Diário Oficial [da] República Federativa do Brasil. Brasilia 06 nov 2002.

6 Silva NC, Nogueira LT. Avaliação de indicadores operacionais de um serviço de atendimento móvel de urgência. Cogitare Enfermagem, [S.I], Jul/Set. 2012 [citado em 2018 set. 13]; 17(3):471-7. Disponivel em: https://revistas.ufpr.br/cogitare/article/ view/29287/19037

7 Tibaes HBB, Silva DM, Alves M, Penna CMM, Brito MJM. Perfil de Atendimento do Serviço de Atendimento Móvel de Urgência no Norte de Minas Gerais. J Res: Fundam Care Online, 2018 [citado em 2017 abr. 09]. Jul./Set. 10(3): 675-82. Disponível em: http://dx.doi.org/10.9789/2175-5361.2018.v10i3.675-682

8 Aminzadeh F, Dalziel WB. Older adults in the emergency department: A systematic review of patterns of use, adverse outcomes, and effectiveness of interventions. Annals of Emergency Medicine. 2002 [citado em 2017 set. 05], $39(3): 238$ - 47. Disponivel em: https://doi.org/10.1067/mem.2002.121523

9 Samaras N. Chevalley T, Samaras D, Gold G. Older Patients in the Emergency Department. Annals of Emergency Medicine. 2010 [citado em 2017 dez. 13]; 3(56):261 - 9. Disponivel em: https://doi.org/10.1016/j.annemergmed.2010.04.015

10 Ministério da Saúde (BR). Portaria no 824, de 24 de junho de 1999. Aprova o texto de normatização de atendimento préhospitalar, constante do anexo desta portaria. Diário oficial [da] república federativa do Brasil. Brasilia 25 jun 1999.

11 Rocha EGA, Morais AC, Benevides TO. Serviço de atendimento móvel de urgência (SAMU) no municipio de Juazeiro (BA): principais especialidades demandadas. Rev Baiana Saúde Pública. 2012 [citado em 2018 jan. 22]; 36(4): 1041-52. Disponível em: http://www.scielo.br/pdf/cadsc/v25nl/1414-462X-cadsc-25-1-51.pdf

12 Pitteri JSM, Monteiro PS. Caracterização do Serviço de Atendimento Móvel de Urgência (SAMU) em Palmas-Tocantins, Brasil, em 2009. Com Ciênc Saúde. 2010 [citado em 2017 nov. 14];21(3):227-36. Disponivel em: http://bvsms.saude.gov.br/bvs/ artigos/caracterizacao_servico_atendimento_movel.pdf

13 O'dwyer G, Konder MT, Reciputti LP, Macedo C, Lopes MGM. O processo de implantação do Serviço de Atendimento Móvel de Urgência no Brasil: estratégias de ação e dimensões estruturais. Cad Saúde Pública [Internet]. 2017[citado em 2017 ago. 07]; 33(7):e00043716. Disponivel em: http://www.scielo.br/scielo.php?script=sci_arttextEpid=S0102$311 \times 20170007050108$ ing $=$ en

14 Luchtemberg MN, Pires DEP. Enfermeiros do Serviço de Atendimento Móvel de Urgência: perfil e atividades desenvolvidas. Rev Bras Enferm [online]. 2016 [citado em 2018 fev. 19]; 2(69):194-201. Disponivel em: http://dx.doi.org/10.1590/0034$7167.2016690202 i$

15 Ministério da Saúde (Brasil). Portaria № 1.600, de 07 de julho de 2011. Reformula a Política Nacional de Atenção às Urgências e institui a Rede de Atenção às Urgências no Sistema Único de Saúde. Diário Oficial [da] República Federativa do Brasil 08 jul 2011

16 Lira FB, Ulle CMS, Mattos M. Acidentes motociclisticos e ações educativas no trânsito em municipio do estado de Mato Grosso. Enferm Foco. 2019 [citado em 2020 mar. 17]; v(3). DOI: https://doi.org/10.21675/2357-707X.2019.v10.n3.2013.

17 Acosta AM, Pelegrini AHW, Lima MADS. Percepção dos profissionais de saúde sobre os usuários frequentes dos serviços de urgência e emergência: revisão integrativa. Enferm Foco. 2011 [citado em 2020 mar. 17]; 2 (2): 141-44. DOI: https://doi. org/10.21675/2357-707X.2011.v2.n2.114. 